\title{
Resultados y aprendizaje de la docencia en tiempos de confinamiento.
}

\author{
Verdejo Gimeno, Pedro ${ }^{a}$ Hilario Pérez, Lucia ${ }^{b}$ \\ ${ }^{a}$ Universidad CEU Cardenal Herrera, Valencia, España, pverdejo@uchceu.es ${ }^{b}$ Universidad CEU \\ Cardenal Herrera, Valencia, España, luciah@uchceu.es.
}

\begin{abstract}
Resumen
Nadie podría imaginarse la situación vivida durante la cuarentena sufrida a raíz de la pandemia mundial del Covid-19, situación que como en todos los diferentes estamentos, modifico de forma sustancial la forma de enseñar $y$ aprender, así como los canales tradicionales de aprendizaje.

Durante este confinamiento se hizo necesario continuar con el proceso de enseñanza-aprendizaje cambiando el aula tradicional por plataformas digitales que permitieran el flujo continuo de comunicación e interacción entre el profesor y el alumno. Además, se debieron de utilizar nuevas herramientas para permitir la evaluación del alumnado, lo que supuso un verdadero reto tanto por la ausencia del alumno físicamente en el proceso, como por establecer un control durante el proceso de evaluación.

Esta gran experiencia, no cabe duda de que realizada en un tiempo record, no ha tenido únicamente inconvenientes, pues también se han podido evidenciar ventajas sustanciales que en gran medida pueden ser trasladadas en convivencia con la docencia tradicional presencial.
\end{abstract}

Palabras clave: Docencia, confinamiento, nuevos escenarios.

\section{Introducción}

Parece ya muy lejana cuando en 1968 se fundo la "Open University"1 en Reino Unido precursora de la formación multimedia, que supuso un empuje a la formación a distancia

\footnotetext{
1 http://www.open.ac.uk
} 
surgida a finales del siglo XIX. ${ }^{2}$ El siguiente gran impulso vendría de la mano del británico Tim Berners-Lee en 1989 con el inicio del World Wide Web, para que a principios del siglo XXI se comience a ver la formación no presencial como un verdadero modelo a tener presente en la enseñanza.

Como resultado, actualmente muchas universidades se han creado bajo un modelo educativo orientado hacia la no presencialidad con una enseñanza $100 \%$ online, incluso las universidades de carácter presencial han fomentado la combinación de técnicas tradicionales con nuevos formatos propios de la oferta no presencial.

La digitalización de la universidad es un tema que se lleva abordando desde hace más de dos décadas (Laviña \& Mengual, 2008) tanto a nivel estratégico como docente, lo que ha supuesto que en un breve espacio de tiempo una de las mayores revoluciones tanto en la propia idiosincrasia de la universidad como un cambio del modelo educativo sin precedentes. Se puede afirmar que no estamos hablando de una tendencia sino de un hecho. Basta con comprobar que en la educación superior no presencial en España ha aumentado un $5 \%$ en el estudio de grados y un $26 \%$ en postgrados, además se estima que en dos años el $50 \%$ de la educación superior se impartirá con metodología 100\% online. (Fundación Telefónica, 2019)

Por tanto, hemos de ser conscientes de que en un futuro más o menos cercano la enseñanza no presencial estará al mismo nivel que la presencial, en un proceso que deberá de tener en cuenta tanto las nuevas técnicas y recursos que ya la hacen posible, como contar con estructuras organizativas distintas a las de la universidad tradicional (García-Peñalvo, 2020).

Pero ¿estamos preparados para adoptar el modelo de docencia no presencial? ¿ha empezado el declive de la docencia presencial? Estas cuestiones han suscitado en estas dos últimas décadas acalorados debates sobre las ventajas e inconvenientes de un tipo u otro de docencia, destacando las enormes reticencias por parte de los profesores universitarios más tradicionales hacia la docencia online. Curiosamente, todo este debate se ha visto enmudecido de forma inmediata por la gran convulsión que ha suscitado la irrupción de la pandemia por el COVID-19 a nivel global, obligando a que 1370 millones los alumnos hayan debido de interrumpir sus clases, casi el $80 \%$ de la población estudiantil, asumiéndose como única posibilidad la docencia no presencial. ${ }^{3}$

\footnotetext{
2 En 1891 en Francia se empezó a impartir clases por correspondencia y en 1914 se funda en Noruega primera institución de educación a distancia

3 Más de 156 millones de estudiantes están fuera de la escuela en América Latina debido al coronavirus, en Noticias ONU, 26 de marzo de 2020. (www.neJustws.un.org)
} 
La Fundación Universitaria CEU, al igual que la mayoría de las universidades, se ha hallado inmersa en un proceso de cambio y adaptación de su docencia presencial a un formato no presencial en tiempos de confinamiento. En esta profunda transformación realizada en un tiempo record, se han sobrepasado en menos de dos meses más de dos décadas de reticencias y recelos tanto de instituciones como de docentes, aun teniendo a favor haberse configurado posiblemente como el fututo paradigma de enseñanza.

Por ello se hace conveniente considerar los principales aspectos y agentes relevantes que han permitido afrontar con aparente éxito en nuestra Universidad ${ }^{4}$, un cambio sustancial en la propia idiosincrasia que ha caracterizado nuestra enseñanza, que estando significativamente alejada del modelo no presencial, ha experimentado un cambio sin precedentes y presumiblemente no tan efímera como cabria pensar inicialmente, como paso previo para poder establecer los próximos retos de la educación en un futuro de entorno volátil, incierto, complejo y ambiguo, o que algunos definen como entorno VUCA. ${ }^{5}$

\section{Principales factores del cambio}

\subsection{La infraestructura tecnológica}

En la actualidad, todas las universidades cuentan con plataformas docentes que permiten tareas de gestión con sus estudiantes. Previo a la pandemia el uso más habitual de estas plataformas era el de repositorio de contenidos, información de la asignatura, comunicación estudiante-profesor, y reflejar los resultados de los actos de evaluación. Tras el paso perturbador de la pandemia, se ha podido comprobar que en un alto porcentaje se estaba infravalorando su capacidad real, permitiendo realizar desde evaluaciones hasta la impartición de docencia síncrona y asíncrona.

Una de las lecciones aprendidas tras estos primeros meses ha sido la de comprobar las tremendas posibilidades que pueden ofrecer estas plataformas para la gestión y labor docente, siendo unas herramientas mucho más cercanas al alumno actual por ser flexibles, activas y dinámicas. Además de las herramientas propias de las universidades, también se ha podido constatar el tremendo potencial de herramientas comerciales como Office 365, que integran todo un mundo de posibilidades en favorecer las técnicas de aprendizaje. Un

\footnotetext{
4 La Universidad CEU San Pablo se ha situado entre las tres universidades más innovadoras del mercado iberoamericano. Fuente: ¿Qué universidades y escuelas de negocio están a la vanguardia?, en elEconomista, del 14/05/2020.

5 El concepto de entorno VUCA comenzó a ser utilizado en los años noventa por los soldados norteamericanos. Se trata de un acrónimo inglés compuesto por cuatro términos: Volatilily, Uncertainty, Complexity y Ambiguity.
} 
dato relevante del uso extendido de este tipo de plataformas se puede ver en los índices de incremento de su utilización, como por ejemplo el paso de casi 560 millones de minutos en reuniones bajo Microsoft Teams a mediados de marzo, a los más de 4000 millones un mes después. (Microsoft, 2020)

Hoy en día existen infinidad de herramientas que posibilitan la labor docente desde la producción de contenidos, su publicación en repositos institucionales, y su impartición desde plataformas de videoconferencia, gestores académicos o escritorios y pizarras virtuales compartidas. (García-Peñalvo, 2019)

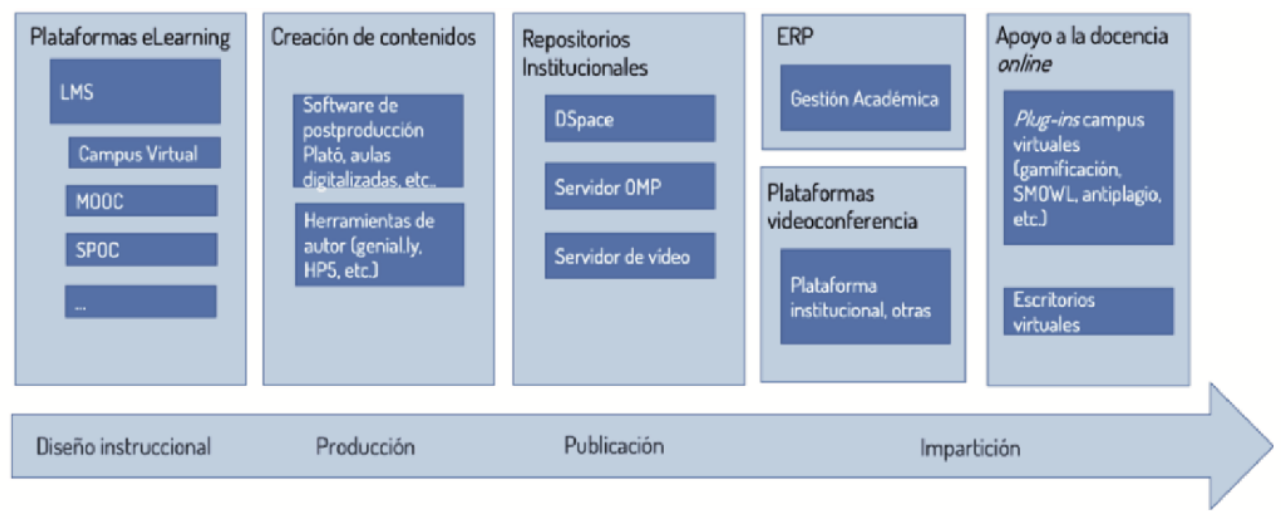

Fig. 1 Esquema del ecosistema tecnológico para la docencia online. Fuente: García-Peñalvo, 2019.

Es decir, los medios e infraestructuras para generar contenido y impartirlos existen, únicamente debemos adecuar las metodologías para usarlas de forma eficaz y favorecer el proceso de enseñanza, pero sin perder o desvirtuar el rol del profesor como guía del aprendizaje.

Por otro lado, aunque la mayoría de las plataformas de gestión docente tienen la posibilidad de realizar diferentes tipos de exámenes o pruebas, la mayoría de ellas carecen de un sistema efectivo de supervisión. Debe entenderse que la supervisión no se tarta tanto de comprobar si un alumno comete actos fraudulentos durante las pruebas, sino por garantizar una evaluación justa, equitativa y que acredite los conocimientos adquiridos por el estudiante. En este apartado, en nuestra Universidad se ha utilizado una novedosa plataforma que posibilita la supervisión a distancia de exámenes ${ }^{6}$, pero aun así, sigue siendo un apartado a recapacitar por las cuantiosas posibilidades que aporta la evaluación en la docencia no presencial, y que pueda adquirir el verdadero sentido de retroalimentar el proceso enseñanza-aprendizaje.

\footnotetext{
${ }^{6}$ Recurso Proctoring de la compañía Mercer | Mettl.
} 


\subsection{El profesor y su nuevo rol}

Esta extensamente definido y asumido el papel del profesor en el acto didáctico que definimos como tradicional y desarrollado en el aula de clase, incluso conocemos desde hace dos décadas las diferencias fundamentales entre las características de la enseñanza tradicional en comparación con el aprendizaje no presencial (Yeung 2001). Pero si atendemos a una de las definiciones de la docencia tradicional, podemos comprobar cuales han sido los cambios sustanciales en el cambio docente. Según Gold (Gold, 2001) la docencia presencial correspondería básicamente a "ensenar a los alumnos un cuerpo de información y conocimientos bien estructurado en un ambiente de aprendizaje bien definido". Obviando el medio de transmisión de los conocimientos, el reto se centra en seguir teniendo unos contenidos estructurados y un ambiente de aprendizaje.

Existen posiciones a favor y en contra sobre cual de las dos modalidades docentes era la más adecuada o ventajosa para favorecer el proceso de enseñanza-aprendizaje, y aunque como se ha indicado anteriormente la docencia on-line esta experimentado un imparable auge, sigue habiendo detractores a un lado y otro. Todo esto se ha visto súbitamente fulminado por condiciones no controlables de nuestro entorno, por lo que unos y otros han tenido que verse abocados a la docencia no presencial.

El pasar de la modalidad presencia a la no presencial, no se basa únicamente en el uso de herramientas tecnologías para posibilitar la docencia sin presencialidad. Significa un cambio profundo del rol del profesor y principalmente, que el docente sea capaz de reunir una serie de competencias y destrezas tecnológicas, didácticas y tutoriales. (Castaño, 2003). Seria muy osado decir que todos los profesores universitarios, independientemente de su relación con la universidad, edad o su recelo a la formación no presencial, reúnan las competencias necesarias para este tipo de formación, existiendo diferentes niveles de adaptación a esta nueva circunstancia. Por ello, la figura del profesor puede haber sido una de las piezas fundamentales y más difícil de adaptar a esta nueva situación docente, principalmente debido al poco tiempo que se ha tenido para favorecer una transición gradual en a los nuevos roles del docente, así como por las históricas reticencias existentes en ciertos casos.

Lejos han quedado otras experiencias sobre la transición gradual desde la docencia convencional a una menor presencialidad para sumergirnos aceleradamente en un cambio en tiempo record, comprobándose en todo caso que independientemente del tiempo de transformación se ha seguido la conocida curva de cambio basados en el modelo de Küber- 
Ross, ${ }^{7}$ siendo clave para hacer efectivo este proceso, el apoyo, aliento y orientación de la institución.

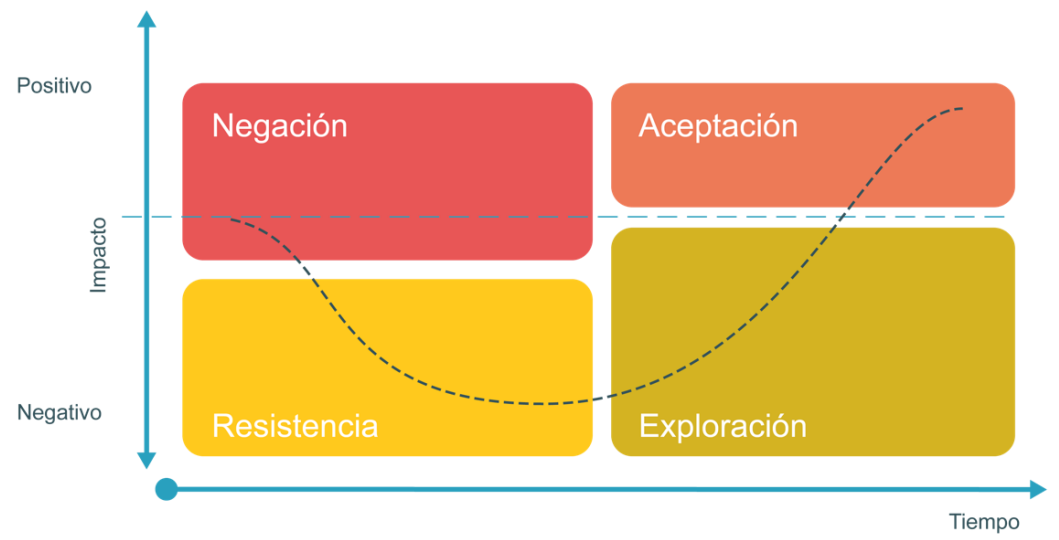

Fig. 3, Esquema del proceso de cambio basado en la curva modelo Kübler-Ross.

Fuente: Fernández, $M$

Tomando como eje narrador la línea temporal del cambio, un pequeño porcentaje de los docentes experimentaron una actitud de negación a adoptar un cambio docente tan sustancial. Es por ello necesario contar con información evidente de la necesidad de cambio, para romper este recelo inicial. Posteriormente se da una fase de resistencia, puede que a lo desconocido o a salir del área de confort del docente, que debe suplirse con formación para favorecer la inserción del docente en una nueva fase de exploración y finalmente de aceptación. Todas estas etapas deben seguir contando con formación, apoyo y orientación en cuanto al uso de técnicas, herramientas y metodologías para hacer eficientes y viable la aceptación del cambio.

Pero sin duda el cambio fue alentado mediante una formación síncrona y asíncrona para los docentes, permitiendo además de formarse, experimentar y compartir experiencias. En este sentido, el hecho de compartir sus propias inquietudes personales, ha permitiendo atender a otro de los aspectos importantes de esta transformación; el profundo cambio a nivel personal y en el rol profesional que el docente experimenta en el tránsito de la enseñanza presencial, conocida y experimentada, a la no presencial o inexplorada.

Es a destacar contrariamente a lo que alertan diferentes investigaciones, a la capacidad de adaptación de los profesores a utilizar la tecnología de manera efectiva en su labor docente. Presumiblemente es debido a que sin darnos cuenta nos encontramos completamente

\footnotetext{
${ }^{7}$ El modelo Kübler-Ross, comúnmente conocido como las cinco etapas del duelo, postula un proceso por el cual la gente lidia con la tragedia. On death and dying, 1969.
} 
sumergidos en la era digital, por lo que es más fácil asimilar los nuevos recursos tecnológicos. Por otro lado, los profesores al igual que los alumnos, con la formación adecuada y con suficiente motivación, pueden ser dotados de las capacidades necesarias para enfrentarse a este nuevo escenario. Como resultado, la valoración del alumnado durante la docencia en el periodo de confinamiento ha sido en gran medida satisfactoria en la labor realizada por sus profesores.

\subsection{Adaptación de contenidos a los nuevos medios de comunicación}

Obviamente, el poco tiempo que se ha tenido para adaptarse al cambio no ha sido suficiente para adecuar completamente los contenidos, pero ha servido para comprobar como los recursos existentes pueden favorecer el aprendizaje. Actualmente, además de las plataformas síncronas de docencia, que serian lo más parecido a la exposición de los contenidos como en una clase magistral, el uso de recursos como genially o mentimeter favorecen la interacción con los estudiantes como clave para que se produzca aprendizaje, colaboración y una participación activa por parte de todos los involucrados en la acción formativa. Además, son herramientas que nos permiten verificar en tiempo real o de forma diferida mediante sondeos, encuestas o la resolución de cuestiones breves, la asimilación de los contenidos expuestos, lo que habilita incluso la corrección o mejora en su uso.

En este sentido, la posibilidad de interacción con los estudiantes a través de componentes asíncronos con flexiblilidad en cuanto acceso y seguimiento, abren una nueva dimensión en cuanto a la formación del estudiante y a su autonomía frente al aprendizaje. Por todo ello, aunque en esta transformación docente se ha dejado a disposición de los estudiantes todos los materiales multimedia propios de una sesión presencial (videos, imágenes, presentaciones en PowerPoint...), debe entenderse que la correcta adecuación que se necesita de los contenidos, no se limita al mero hecho de poner en alguna de las plataformas de repositorio todos los contenidos empleados (Gallego, 2008). Es necesario un rediseño de los contenidos y adecuarlos correctamente a las diferentes posibilidades que nos brindan los recursos de la enseñanza no presencial dirigidos al autoaprendizaje del estudiante. Incluso, en el supuesto de continuar con la docencia presencial, el hecho de contar con todos estos recursos, supone un salto significativo en el aprendizaje autónomo del estudiante.

\section{El reto de la docencia en la "nueva normalidad"}

Ante un futuro volátil y un entorno incierto, el hecho de que una Universidad que ha desarrollado su actividad educativa desde 1933 bajo el paradigma presencial, verse abocada 
a un cambio tan sustancial hacia un modelo no presencial por la necesidad inapelable de un estado de emergencia, no es sinónimo de fracaso. Muestra de ello es estar valorada recientemente entre las primeras universidades más innovadoras del mercado iberoamericano.

Los cambios, sobre todo en un modelo docente de gran tradición y que forman parte de la propia idiosincrasia que caracterizan una universidad, siempre generan rechazo y reticencias, pero en estos tiempos convulsos, no hay otro camino que superarlos dando ejemplo a nuestros estudiantes de innovación, flexibilidad y adaptación al cambio.

Finalizado esta perturbación con todos sus inconvenientes y ante la incerteza de asegurar la continuidad de la docencia presencial en un futuro cercano, se debe hacer una reflexión profunda sobre las lecciones aprendidas durante este confinamiento, intentando mejorar el proceso de enseñanza y aprendizaje con todos los medios y herramientas a nuestro alcance.

Parece por ello lógico, que todas las ventajas que ha aportado la adopción de la metodología de docencia online, puedan ser absorbidas para implementar la presencial y hacerla más efectiva, como para tener la posibilidad de generar un modelo semipresencial ante futuros escenarios volátiles. Los recusos necesarios existen, lo que se hace necesario es rediseñar los contenidos, adecuarlos a los recursos tecnológicos, formar al profesorado en la adquisición de competencias específicas, y optimizar una evaluación formativa.

Cabe decir que el recuso que más controcersia ha suscitado ha sido el de control y supervisión de las pruebas evaluatorias. Por un lado, ya que se tuvieron que hacer, tanto por profesores como por alumnos, pruebas preparatorias para poder contemplar todos los posibles escenarios, debido a que gran numero de los estudiantes son de origen internacional y estaban en sus países de origen. Por otro lado, el profesor ha debido de reflexionar de como trasnformar el tradicional examen al modelo no presencial, existiendo en este caso infinidad de casuísticas, debido a la diversidad de materias y contenidos. Aún así, en todos los casos se ha podido asegurar una evaluación justa, equitativa y que ha acreditado los conocimientos adquiridos por el estudiante.

Aunque los alumnos han valorado muy positivamente el esfuerzo realizado por el profesorado y la Institución, la rapidez de adaptación y la disposición del profesorado, cabe decir que el estudiante, menos reticente al uso de toda la tecnología y recursos, ya no espera una "docencia de supervivencia", sino que entiende que la universidad y toda su estructura debe estar comprometida para asegurar la continuidad de su formación tanto en calidad como ante un futuro confuso. 


\section{Referencias}

Castaño, C. (2003): 1 rol del profesor en la transici $n$ de la ense an a presencial al aprendi a je on line». o municar 1 e vista ien $\mathrm{t}$ fica de o municaci $\mathrm{n} \mathrm{y}$ d ucaci $\mathrm{n}$; $\mathrm{p}$ ginas 9-55. Consultado en https://www.redalyc.org/pdf/158/15802107.pdf

Fundación Telefónica. (2019): Sociedad digital en España 2018. Barcelona, Spain: Penguin Random house grupo Editorial. Consultado en www.electrónicaadministracionelectronica.gob.es

Jiménez, A.M. (2009): La escuela nueva y los espacios para educar. Revista Educación y Pedagogía, (54), pp. 103-125. Consultado en www.dialnet.unirioja.es

Gallego 8 : omunicaci $\mathrm{n}$ did ctica del docente universitario en entornos presenciales y virtuales en e vista eroamericana de d ucaci $\mathrm{n} n$ 61. Consultado en https://rieoei.org/RIE/article/view/2017

García-Peñalvo, f. J. (2020): Modelo de referencia para la enseñanza no presencial en universidades presenciales. Campus Virtuales, 9(1), 41-56. Consultado en https://repositorio.grial.eu/handle/grial/1988

García-Peñalvo, f. J. (2019): Modelo de docencia Virtual para una Universidad Presencial. En IX Jornadas Internacionales de Campus Virtuales. Consultado en https://repositorio.grial.eu/bitstream/grial/1703/1/CampusVirtuales2019.pdf

Gold, S. (2001): A constructivist approach to on-line training for on-line teachers, en Journal of Asynchronous Learning Networks, 5 (1); 35-57. Consulatdo en www.onlinelearningconsortium.org

Yeung, D. (2001): Quality assurance of web-based learning in distance education institutions, en Journal of Distance Learning Administration, 4. Consult from www.westga.edu/ distance/ojdla/winter- 44/yeung44.html 